\title{
WIENER-HOPF TYPE PROBLEMS FOR ELLIPTIC SYSTEMS OF SINGULAR INTEGRAL EQUATIONS ${ }^{1}$
}

\author{
BY ELIAHU SHAMIR
}

\section{Communicated by F. Browder, February 2, 1966}

The problem treated in this paper is roughly the inversion of elliptic systems of singular integral equations in a half-space of $R^{n}$. Ellipticity means that the system is invertible over the whole of $R^{n}$, in our case explicitly. We first introduce notation and some spaces of (vector-valued) distributions.

Let $(x, y)$ denote points in $R^{n}$ with $x \in R^{n-1}, y \in R . R_{+}^{n}\left[R_{-}^{n}\right]$ is the half-space $y \geqq 0[y \leqq 0] . H^{s, p}$ is the space of distributions $u$ for which

$$
\|u\|_{s, p}=\left\|F^{-1}\left(1+|\xi|^{2}+\eta^{2}\right)^{s / 2} F u\right\|_{L^{p}}<\infty .
$$

Here $(F u)(\xi, \eta)=\int u(x, y) e^{i(x \cdot \xi+y \cdot \eta)} d x d y$, with $(\xi, \eta)$ dual to $(x, y)$. We assume $1<p<\infty . H_{-}^{s, p}$ is the subspace of elements supported in $R_{-}^{n}$. $H^{s, p}\left(R_{+}^{n}\right)$ is the quotient $H^{s, p} / H_{-}^{s, p}$ (it is a space of distributions on $\stackrel{\circ}{R}_{+}^{n}$, the open half-space). $Y_{+}$denotes the canonical map onto the quotient and $\left\|Y_{+} u\right\|_{s, p}$ is the quotient norm. $H_{+}^{s, p}, H^{s, p}\left(R_{-}^{n}\right)$ and $Y_{-}$ are similarly defined. For $s=0$, we can identify $H_{ \pm}^{0, p}=L_{ \pm}^{p}$ with $L^{p}\left(R_{ \pm}^{n}\right)$, and $Y_{ \pm}$with multiplication by the characteristic function of $R_{ \pm}^{n}$. The definitions above extend to vector valued functions component-wise.

Let $M(\xi, \eta)$ be an $N \times N$ matrix of functions, positively homogeneous of degree $0, C^{l+1}$ on $|\xi|^{2}+\eta^{2}=1$ where $l>n / 2$. The operator $\boldsymbol{M}=F^{-1} M(\xi, \eta) F$ (whose symbol is $M(\xi, \eta)$ ) is bounded in $H^{s, p}$, invertible (elliptic) if $\operatorname{det}[M(\xi, \eta)] \neq 0$ for $(\xi, \eta) \neq 0$.

THEOREM A. The operator $\tilde{M}: u \rightarrow\left(Y_{-} u, Y_{+} M u\right)$ has a closed range in $H^{s, p}\left(R_{-}^{n}\right) \times H^{s, p}\left(R_{+}^{n}\right)$ for every $s$ except at most $N$ exceptional values of $s(\bmod 1)$. There exists $k^{\prime} \geqq k^{\prime \prime}$ such that for $s=k+\sigma$ nonexceptional

$$
\begin{gathered}
\|u\|_{s, p} \leqq C\left[\left\|Y_{-} u\right\|_{s, p}+\left\|Y_{+} M u\right\|_{s, p}\right], \quad \text { all } u \in H^{s, p} ; k \geqq k^{\prime}, \\
\sum_{ \pm}\left\|V_{ \pm}\right\|_{-s, p^{\prime}} \leqq C\left\|V_{-}+M V_{+}\right\|_{-s, p^{\prime}}, \quad \text { all } V_{ \pm} \in H_{ \pm}^{-s, p^{\prime}} k \leqq k^{\prime \prime} ; \\
k^{\prime \prime}=k^{\prime} \text { in the scalar case. }
\end{gathered}
$$

The first estimate means that $\tilde{M}$ is $1-1$ and has a closed range. The second ("dual") estimate assures that the range of $\tilde{M}$ is full. Thus as $s \rightarrow+\infty$ the operator $\tilde{M}$ becomes left invertible, as $s \rightarrow-\infty$ it becomes right invertible.

1 This work was supported in part by grant GP-3940 from the National Science Foundation. 
We proved those estimates before for $n=1$ and general $M$ [6], or $M=I$ (the identity matrix) and general $n$ [7]. In the latter case the exceptional values are $s=1 / p(\bmod 1)$. (This is so whenever $M(0,1)=M(0,-1)$.) In fact, Theorem A shows that $\tilde{M}$ for general $M$ behaves pretty much like $\tilde{I}$.

Theorem A follows from:

THEOREM B. The operator $Y_{+} u \rightarrow Y_{+} M Y_{+} u$ has a closed range in $L^{p}\left(R_{+}^{n}\right)$ if and only if the eigenvalues of $M(0,-1) \cdot M^{-1}(0,1)$ do not lie on the ray $\arg \lambda=2 \pi / p$. Let

$$
M_{k}(\xi, \eta)=(\eta-i|\xi|)^{k} M(\xi, \eta)(\eta+i|\xi|)^{-k} .
$$

There are integers $k^{\prime}, k^{\prime \prime}$ such that $Y_{+} M_{8} Y_{+}$is 1-1 if $k \geqq k^{\prime}$, onto if $k \leqq k^{\prime \prime}$ (provided $Y_{+} M Y_{+}$has a closed range).

REMARK. The estimates of Theorem A settle the problem of obtaining a priori $L^{p}$ estimates for elliptic partial differential equations in $n+1$ dimensional domains with piecewise smooth boundary operators (so called "mixed" problems). These (usually $L^{2}$ ) estimates were obtained before under very special conditions [3], [4], [5] and $[8]$.

Method of Proof. For Theorem B, we first prove the following reduction:

Lemma 1. Let $M_{\bar{\xi}}=F^{-1} M\left(\bar{\xi}, \eta|\xi|^{-1}\right) F$. Then estimate

$$
\left\|Y_{+} u\right\| \leqq C\left\|Y_{+} M Y_{+} u\right\|, \quad u \in L^{p}
$$

(norms are $L^{p}\left(R_{+}^{n}\right)$ norms) is equivalent to the family of estimates

$$
\left\|Y_{+} u\right\| \leqq C\left\|Y_{+} M_{\bar{\xi}} Y_{+} u\right\|, \quad u \in L^{p}
$$

for all $\bar{\xi}$ satisfying $|\bar{\xi}|=1$.

The operators $M_{\bar{\xi}}$ are easier to study since their symbols are essentially one-dimensional. As usual with this type of problem, one tries to factor the symbol into product of matrices holomorphic in $\operatorname{Im} \eta>0$ and $\operatorname{Im} \eta<0$. Here it suffices to factor $M(\bar{\xi}, \eta)$ for fixed $\bar{\xi}$ and then substitute $\eta|\xi|^{-1}$ for $\eta$. We use results of Gohberg-Krein [1], [2]. They factor matrices of the form $I+K(\eta)$ where $K(\eta) \in F L^{1}$ (or a suitable subring, cf. [1]). Thus we first have to "fill in" the jump of $M(\bar{\xi}, \eta)$ at infinity in case $M(0,1)=M(\xi, \infty) \neq M(\xi,-\infty)=M(0,-1)$. If the jump matrix

$$
M(0,-1) \cdot M^{-1}(0,-1) \text { is similar to } \operatorname{diag}\left[\lambda_{1}, \cdots, \lambda_{N}\right],
$$

this is readily done by diagonal factors of the form 


$$
(\eta \pm i)^{\sigma}=\operatorname{diag}\left[(\eta \pm i)^{\sigma}, \cdots,(\eta \pm i)^{\sigma}\right]
$$

where $\sigma=\left(\sigma_{1}, \cdots, \sigma_{N}\right)$ is $N$-tuple of fractional (may be complex) numbers determined by $\lambda_{1}, \cdots, \lambda_{N}$. Indeed $M_{1}(\bar{\xi}, \eta)=(\eta-i)^{-\sigma}$ $M(\bar{\xi}, \eta)(\eta+i)^{\sigma}$ has the same value at $\pm \infty$ and is factorizable. Factoring it, we get for $M(\bar{\xi}, \eta)$

Lemma 2. For a fixed $\bar{\xi} \neq 0$ we have (suppressing the dependence on $\bar{\xi})$ :

$$
M(\xi, \eta)=Q_{-}^{-1}(\eta)(\eta-i)^{\sigma}\left(\frac{\eta-i}{\eta+i}\right)^{k}(\eta+i)^{-\sigma} Q_{+}(\eta)
$$

where $Q_{+}(\eta)\left[Q_{-}(\eta)\right]$ and its inverse are bounded and smooth for real $\eta$, have holomorphic extension to $\operatorname{Im} \eta>0$ [Im $\eta<0]$. Moreover, their derivatives decrease as $|\eta| \rightarrow \infty$ in a manner which assures that $Q_{ \pm}\left(\eta|\xi|^{-1}\right)$ and their inverses are $L^{p}$-multipliers. $\kappa=\left(\kappa_{1}, \cdots, \kappa_{N}\right)$ is a nonincreasing sequence of integers which are uniformly bounded for $|\bar{\xi}|=1$.

REMARK. If (2) is not satisfied, the factorization of $M$ is more complicated but the final results remain unchanged.

Lemma 3. Let $Q_{ \pm}=F^{-1} Q_{ \pm}\left(\eta|\xi|^{-1}\right) F$ and

$$
D_{\kappa+\sigma}=F^{-1}(\eta-i|\xi|)^{\sigma}\left(\frac{\eta-i|\xi|}{\eta+i|\xi|}\right)^{\sigma}(\eta+i|\xi|)^{-\sigma} F .
$$

Then $Q_{+}$sets an isomorphism between the null-spaces of $Y_{+} M_{\bar{\xi}} Y_{+}$and $Y_{+} D_{k+\sigma} Y_{+} Q_{-}$sets an isomorphism between the ranges of these operators in $L^{p}\left(R_{+}^{n}\right)$. have

Notice that $D_{\kappa+\sigma}$ is a direct sum of scalar operators for which we

Lemma 4. If $k$ is an integer and $-1+1 / p<\operatorname{Re} \sigma \leqq 1 / p$ then the (scalar) operator $Y_{+} D_{k+\sigma} Y_{+}$has a closed range if and only if $\operatorname{Re} \sigma \neq 1 / p$. In this case it is $1-1$ if $k \geqq 0$, onto if $k \leqq 0$.

The proof of Theorem B follows now quite easily from Lemmata 1-4; for Theorem A we need two more. Let us denote

$$
J_{ \pm}^{t}=F^{-1}\left[\eta \pm i\left(1+|\xi|^{2}\right)^{1 / 2}\right]^{t} F \text {. }
$$

Lemma 5. $J_{-}^{t}$ maps $H^{s, p}\left(R_{+}^{n}\right)$ onto $H^{s-t, p}\left(R_{+}^{n}\right)$ (in particular onto $L^{p}\left(R_{+}^{n}\right)$ if $s=t$ ) and $\left\|Y_{+} J_{-}^{t} u\right\|_{8-t, p} \sim\left\|Y_{+} u\right\|_{8}$. (Similar statement with + and - interchanged.)

LEMMA 6. The following estimates are equivalent 


$$
\begin{aligned}
& \|u\|_{s, p} \leqq C\left[\left\|Y_{-} u\right\|_{s, p}+\left\|Y_{+} M u\right\|_{s, p}\right], \quad u \in H^{s, p} \text {, } \\
& \|v\|_{0, p} \leqq C\left[\left\|Y_{-} v\right\|_{0, p}+\left\|Y_{+} J_{-}^{s} M J_{+}^{-8} v\right\|_{0, p}\right], \quad v \in L^{p} \text {, } \\
& \left\|Y_{+} v\right\|_{0, p} \leqq C\left\|Y_{+} M_{8} Y_{+} v\right\|, \quad v \in L^{p} \quad \text { (cf. (1)), } \\
& \sum_{ \pm}\left\|V_{ \pm}\right\|_{-8, p^{\prime}} \leqq C\left\|V_{-}+M V_{+}\right\|_{-s, p^{\prime}}, V_{ \pm} \in H^{-8, p^{\prime}} \text {. }
\end{aligned}
$$

\section{BIBLIOGRAPHY}

1. I. C. Gohberg, Factorization problems in normed rings, Uspehi Mat. Nauk 19 (1964) 71-124.

2. I. C. Gohberg and M. G. Krein, Systems of singular integral equations in half line, Uspehi Mat. Nauk 13 (1958), 3-72.

3. J. Peetre, Mixed problems for higher order elliptic equations in two dimensions. I, Ann. Scuola Norm. Sup. Pisa (3) 15 (1961), 337-353; II, Ann. Scuola Norm. Sup. Pisa (3) 17 (1963), 1-12.

4. M. Schechter, Mixed problems for higher order elliptic partial differential equations, Comm. Pure Appl. Math. 13 (1960), 407-425.

5. E. Shamir, Mixed boundary value problems for elliptic equations in the plane. The $\mathrm{L}_{p}$ theory, Ann. Scuola Norm. Sup. Pisa (3) 17 (1963), 117-138.

6. - Reduced Hilbert transforms and singular integral equations, J. Analyse Math. 12 (1964), 227-305.

7. - Une propriêté des espaces $\mathrm{H}^{s, p}, \mathrm{C} . \mathrm{R}$. Acad. Sci. Paris, 255 (1962), 448-449.

8. I. M. Višik and G. I. Esskin, General boundary value problems with discontinuous conditions at the boundary, Dokl. Akad. Nauk SSSR 158 (1964),15 = Soviet Math. Dokl. 5 (1964), 1154.

NORTHWESTERN UNIVERSITY 